\title{
Produção de tecnologia educacional como estratégia de prevenção de LER e DORT's
}

\author{
Production of educational technology as a strategy for the prevention of RSI and WMSD \\ Producción de tecnología educativa como estrategia para la prevención de RSI y WMSD
}

lana Sá Silva ${ }^{1 *}$, Alex de Freitas Marques ${ }^{1}$, José Duban Tabarquino Vargas ${ }^{1}$, Marina Silva Nicolau Taketomi ${ }^{1}$.

\section{RESUMO}

Objetivo: Construir e validar uma Tecnologia Educativa do tipo papel bandeja para a prevenção de doenças ocupacionais. Métodos: Foi realizada uma pesquisa quantitativa de validação de tecnologia educativa, o material construído foi do tipo papel bandeja com orientações sobre ergonomia no ambiente do trabalho, o público alvo foram os funcionários do setor operacional de uma empresa de processamento de grãos no município de em Santarém - Pará, o material passou por quatro etapas de construção e validação e foi avaliado por dois grupos de juízes: os funcionários da empresa e os fisioterapeutas especialistas, através da escala de Likert. Resultados: A tecnologia elaborada e composta por uma lauda e atingiu $87,5 \%$ de concordância dos avaliadores especialistas e 100\% dos beneficiários para validação segundo a escala de Likert. Conclusão: A tecnologia educativa elaborada foi considerada válida para utilizar com o público-alvo e atingiu o objetivo de orientações de alongamentos diários e ergonomia, sendo considerada de fácil acesso e entendimento.

Palavras-chave: Tecnologia educativa, Saúde do trabalhador, Ergonomia.

\begin{abstract}
Objective: To build and validate an Educational Technology of the paper tray type for the prevention of occupational diseases. Methods: A quantitative research was carried out to validate educational technology, the material constructed was of the paper tray type with guidelines on ergonomics in the work environment, the target audience was the employees of the operational sector of a grain processing company in the municipality of em Santarém - Pará, the material went through four stages of construction and validation and was evaluated by two groups of judges: company employees and specialist physiotherapists, using the Likert scale. Results: The technology elaborated and composed by a page and reached $87.5 \%$ of agreement of the expert evaluators and $100 \%$ of the beneficiaries for validation according to the Likert scale. Conclusion: The educational technology developed was considered valid for use with the target audience and reached the goal of daily stretching and ergonomics guidelines, being considered easy to access and understand.
\end{abstract}

Key words: Educational technology, Occupational health, Ergonomics.

\section{RESUMEN}

Objetivo: Construir y validar una Tecnología Educativa del tipo bandeja de papel para la prevención de enfermedades laborales. Métodos: Se realizó una investigación cuantitativa para validar tecnología educativa, el material construido fue del tipo bandeja de papel con lineamientos en ergonomía en el ambiente de trabajo, el público objetivo fueron los empleados del sector operativo de una empresa procesadora de granos en el municipio de En Santarém - Pará, el material pasó por cuatro etapas de construcción y validación y fue evaluado por dos grupos de jueces: empleados de la empresa y fisioterapeutas especialistas, utilizando la escala Likert. Resultados: La tecnología elaborada y compuesta por una página y alcanzó el $87,5 \%$ de acuerdo de los evaluadores expertos y el $100 \%$ de los beneficiarios para su validación según la escala Likert. Conclusión: La tecnología educativa desarrollada se consideró válida para su uso con el público objetivo y alcanzó el objetivo de pautas de estiramiento diario y ergonomía, considerándose de fácil acceso y comprensión.

Palabras clave: Tecnología educación, Salud del trabajo, Ergonomia.

\footnotetext{
${ }^{1}$ Instituto Esperança de Ensino Superior (IESPES), Santarém - PA. *E-mail: iana.sa.silva@gmail.com
} 


\section{INTRODUÇÃO}

Nas últimas décadas vem crescendo gradativamente o número de incidência de dor e lesão muscular relacionada ao trabalho, sendo essas as mais prevalentes causas de afastamento, ausência e incapacidade no ambiente de trabalho. Essas queixas são conhecidas como LER/DORT (Lesão por Esforço Repetitivo/ Distúrbio Osteomuscular Relacionado ao Trabalho), e abrangem diretamente a produtividade e experiência profissional gerando ausências temporárias, repetitivas e até mesmo definitivas (HUGUE TD e PEREIRA AA, 2011).

Neste contexto, a prevenção é o meio mais indicado de minimizar prejuízos à saúde do trabalhador e gastos financeiros advindos em razão do processo de adoecimento (SERRA MVGB, et al., 2014). Deste modo, visando alcançar a melhora da saúde laboral, tornou-se cada vez mais importante a inclusão do fisioterapeuta no meio industrial e empresarial, objetivando promover a qualidade de vida do trabalhador e prevenir lesões musculoesqueléticas. O efeito desta intervenção é a melhora no desempenho e na produtividade no trabalho. Atuando na implantação de programas de ergonomia, de cinesioterapia laboral, e reabilitando trabalhadores afastados por LER/DORT, trazendo uma melhoria no rendimento da empresa, e um melhor bem-estar físico e social para seus colaboradores (MELO IA, 2017).

Uma Tecnologia Educativa (TE) caracteriza-se por ferramentas capazes de mediar o processo de ensino e aprendizagem, utilizada entre educadores (profissionais de saúde) e educandos (clientela) nas diferentes metodologias de educação, seja acadêmica ou continuada, proporcionando ao educando e educador uma construção e reconstrução do conhecimento, contribuindo para um avanço educacional em saúde (MELO IA, 2017; MOREIRA AP, et al., 2014; NIETSCHE EA, et al., 2012; NASCIMENTO MHM, 2012).

Posto isso, com a crescente nos índices de afastamento laboral em decorrência de doenças ocupacionais, a educação em saúde assume um papel de destaque neste cenário, viabilizando em primordial a conscientização e a modificação de hábitos. Desta forma, a pesquisa se propôs em construir e validar uma TE do tipo papel bandeja para a prevenção de doenças ocupacionais em uma empresa privada de processamento de grãos no município de Santarém - Pará.

\section{MÉTODOS}

Desenvolveu-se uma pesquisa metodológica quantitativa de validação de TE. Este modelo de pesquisa busca o desenvolvimento, avaliação e aperfeiçoamento de instrumentos e estratégias metodológicas (MONTEIRO DS, et al., 2019). A tecnologia validada nesse estudo foi do tipo papel bandeja com orientações sobre alongamentos e ergonomia para funcionários da equipe de produção de uma empresa de processamento de grãos em Santarém-PA. O processo de validação da TE foi dividido em 4 etapas, sendo elas: $1^{\underline{a}}$ etapa - entrevista com os funcionários; $2^{\underline{a}}$ - etapa elaboração da TE de acordo com o que foi solicitado; $3^{3}$ - etapa julgamento da TE pelos juízes laborais e beneficiários; $4^{a}$ - etapa entrega do material para empresa.

Para a elaboração da TE foi realizado inicialmente uma pesquisa de campo laboral com a empresa escolhida para a execução do estudo, onde após, a assinatura do Termo de Consentimento Livre Esclarecido (TCLE), a ferramenta utilizada para coleta de dados foi um questionário semiestruturado aplicado ao público alvo (funcionários da equipe de produção) focando nos seguintes questionamentos: dados sociodemográficos (idade, sexo, escolaridade), questões referentes ao trabalho (tempo de empresa, tipos de tarefa realizadas, rotatividade no trabalho), conhecimento sobre LER/DORT's (sabe o que é LER/DORT's, já se afastou do trabalho por algum motivo, utilização de Equipamentos de Proteção Individual (EPI), se relata alguma dor).

No final do questionário os funcionários poderiam dar sugestões sobre assuntos que eles gostariam que fossem abordados no material educativo. Após a aplicação do questionário, totalizando 16 funcionários, evoluiu para elaboração da TE que foi desenvolvida de acordo com a solicitação realizada dos mesmos. Depois de produzida a TE, os juízes laborais e juízes beneficiários receberam o convite para participação da pesquisa de forma voluntária, seguindo o TCLE para assinatura. A pesquisa contou com 4 juízes laborais (especialistas) sendo eles profissionais fisioterapeutas com experiências na área de saúde do trabalhador e 4 juízes beneficiários (funcionários), sendo esses os trabalhadores da empresa onde a pesquisa foi desenvolvida, os quais foram selecionados de forma aleatória para avaliação da TE. 
O instrumento utilizado com juízes laborais e juízes beneficiários para avaliação da TE foi à escala Likert, adaptada para este estudo a partir de sua aplicação em pesquisa de validação, entendendo-se a necessidade da utilização de instrumento sistematizado capaz de mensurar respostas de maneira apropriada.

A escala Likert é uma lista de classificação composta por itens que manifestam avaliação positiva ou negativa sobre um determinado tópico. O julgamento foi feito de acordo com uma pontuação de respostas que variaram com valores numéricos e sua avaliação. Assim o valor 1 TA (totalmente adequado) e $2 \mathrm{~A}$ (adequado) indicavam alta aceitabilidade, os valores 3 PA (parcialmente adequado) e 4 I (inadequado) indicavam média e baixa aceitabilidade.

A escala para os juízes laborais foi composta por 3 itens divididos em 3 blocos sendo organizados em: Objetivo, Estrutura/Apresentação e Relevância. A dos juízes beneficiários foi instituída por 5 itens e fracionada em 5 blocos sendo eles: Objetivo, Organização, Aparência, Estilo de escrita e Motivação.

O bloco Objetivo trazia perguntas pertencentes aos propósitos e metas a atingir com a utilização da tecnologia. Os blocos Estrutura/Apresentação/ Organização ligaram-se à forma de apresentar as orientações, incluindo a organização geral, estrutura, estratégia de apresentação, coerência e formatação.

A Relevância tratava das características que avaliam o grau de significância do material. Aparência abordava respeito à clareza do conteúdo em relação à forma como se apresenta. O estilo de escrita considerou as características linguísticas, compreensão e estilo e a Motivação referiu-se à capacidade do material em causar impacto e/ou interesse a quem se destina.

No final da escala havia espaço para alusões e os participantes foram orientados a sugerir com orientações e recomendações que julgassem necessárias, de acordo com seus conhecimentos e experiências. Os dados foram analisados estatisticamente de forma descritiva por meio do software Microsoft Office Excel 2016. Onde foram analisados de forma quantitativa, transcrevendo parâmetros estatísticos, tais como, média, moda, mediana frequência, amplitude, variância e desvio padrão.

Considerou-se os preceitos éticos da Resolução 466/2012 do Conselho Nacional de Saúde do Brasil. Comitê de Ética e Pesquisa da Universidade do Estado Pará, através da Plataforma Brasil, CAAE: 30189520.5.0000.5168e todos os participantes assinaram o Termo de Consentimento Livre e Esclarecido para manifestar sua anuência por escrito.

\section{RESULTADOS}

A Tecnologia educativa validada nesse trabalho, compôs-se por 1 lauda, a qual abordava orientações sobre alongamentos dividido em 3 partes totalizando 11 conteúdos e ergonomia composto por 6 ideias. As informações utilizadas na elaboração TE seguiram a solicitação dos funcionários, após a aplicabilidade do questionário sociodemográfico, sendo executado na primeira etapa do trabalho (Tabela 1).

Tabela 1 - Perfil sociodemográfico dos funcionários da equipe de produção.

\begin{tabular}{ccc}
\hline \multicolumn{3}{c}{ Questionário Sociodemográfico } \\
\hline Sexo & \multicolumn{2}{c}{$100 \%$ masculino } \\
\hline Tempo de serviço & Média: 5 anos & 6 meses \\
\hline Conhece LER/DORT'S & Sim: $68,75 \%$ & Não: $31,25 \%$ \\
\hline Sugestões & Alongamentos, atividade laboral, ergonomia \\
\hline
\end{tabular}

Fonte: Silva IS, et al., 2021.

Para a forma escrita, utilizaram-se textos explicativos sobre o conteúdo. As ilustrações, realizadas por um design gráfico, estavam de acordo com a ideia solicitada pelos funcionários da empresa, cada tópico abordado na tecnologia e estavam dispostas segundo o tema apresentado. 
Após o processo de elaboração da tecnologia educativa, procedeu-se à validação aparente e de conteúdo, realizada por 8 juízes, sendo: quatro juízes especialistas (fisioterapeutas), a idade variou entre 30 a 35 anos, predomínio no sexo masculino, com a variedade de 2 a 5 anos de experiência na área de saúde do trabalhador, sendo suas especializações 1 Doutor e 3 Mestres (Tabela 2).

Tabela 2 - Perfil dos juízes especialistas, n=04.

\begin{tabular}{ccccccc}
\hline Código & Sexo & Idade & Formação & Cargo & $\begin{array}{c}\text { Tempo de } \\
\text { Formação }\end{array}$ & $\begin{array}{c}\text { Tempo Experiência } \\
\text { na Área de Saúde } \\
\text { do Trabalhador }\end{array}$ \\
\hline JE1 & M & 35 & Fisioterapia & Fisioterapeuta / docente & 11 anos & 5 anos \\
JE2 & M & 30 & Fisioterapia & Docente & 8 anos & 4 anos \\
JE3 & M & 35 & Fisioterapia & Fisioterapeuta / docente & 6 anos & 4 anos \\
JE4 & M & 33 & Fisioterapia & Fisioterapeuta / docente & 7 anos & 2 anos \\
\hline \multicolumn{7}{c}{ Média } \\
\hline \multicolumn{7}{c}{ Mediana } \\
\hline
\end{tabular}

Fonte: Silva IS, et al., 2021.

E quatro juízes beneficiários (funcionários), a idade entre 25 a 38 anos com predomínio do sexo masculino, sendo todos funcionários da área de produção da empresa, com experiências entre 3 a 7 anos de serviço (Tabela 3).

Tabela 3 - Perfil dos juízes beneficiários, $\mathrm{n}=04$.

\begin{tabular}{cccccc}
\hline Código & Sexo & Idade & Escolaridade & Ocupação & $\begin{array}{c}\text { Tempo de } \\
\text { Serviço }\end{array}$ \\
\hline JB1 & M & 38 & Ensino médio completo & Operador 2 - Carregadeira & 3 anos \\
JB2 & M & 28 & Ensino médio completo & Operador 1 - Produção & 7 anos \\
JB2 & M & 25 & Ensino médio completo & Operador 2 - Carregadeira & 4 anos \\
JB4 & M & 34 & Ensino Fundamental Completo & Operador 1-Produção & 5 anos
\end{tabular}

Fonte: Silva IS, et al., 2021.

A validação da tecnologia educativa deu-se através do cálculo do Índice de Validade de Conteúdo (IVC). $\mathrm{Na}$ maioria dos itens, encontrou-se o IVC de, pelo menos, 0,75. Deve-se ressaltar que apenas dois itens, 2.7 e 2.9 na avaliação dos juízes especialistas, relativos à escrita utilizada e ao tamanho do título apresentaram valores de IVC abaixo da concordância mínima adotada.

No entanto, calculando-se a média do IVC para cada um dos aspectos avaliativos da cartilha, obteve-se um IVC superior a 0,70. Encontrou-se como média global do IVC o valor de $93,5 \%$, ratificando a validação da aparência e conteúdo junto aos especialistas de $87,5 \%$ e juízes beneficiários 100\% (Tabela 4 e Tabela 5). Mesmo atingindo o IVC mínimo na primeira rodada, foi realizada a segunda rodada de avaliação pelos juízes especialistas, afim de, apresentar as alterações sugeridas por eles na versão inicial. Após a segunda análise foi atingido o valor de validação de $100 \%$. 
Tabela 4 - Tabela das respostas de juízes especialistas por bloco de avaliação.

\begin{tabular}{|c|c|c|c|c|c|c|}
\hline \multirow{3}{*}{ Blocos } & \multirow{3}{*}{ Itens } & \multicolumn{4}{|c|}{ Escores $(\mathrm{N}=04)$} & \multirow{3}{*}{$\begin{array}{c}\text { Índice de } \\
\text { concordância por } \\
\text { item TA+A X } \\
\text { 100/Total de juízes }\end{array}$} \\
\hline & & \multicolumn{4}{|c|}{ Escore $x 100 / T A+A+P A+I=$ Percentual por escore } & \\
\hline & & TA & A & PA & $\mathbf{I}$ & \\
\hline \multirow{7}{*}{ Bloco I } & 1.1 & 2 & 1 & 1 & 0 & 0,75 \\
\hline & 1.2 & 3 & 1 & 0 & 0 & 0,100 \\
\hline & 1.3 & 1 & 3 & 0 & 0 & 0,100 \\
\hline & 1.4 & 2 & 2 & 0 & 0 & 0,100 \\
\hline & 1.5 & 2 & 1 & 1 & 0 & 0,75 \\
\hline & Subtotal & 8 & 7 & 1 & 0 & - \\
\hline & $\begin{array}{l}\text { Percentual } \\
\text { por bloco }\end{array}$ & \multicolumn{5}{|c|}{$90 \%$} \\
\hline \multirow{14}{*}{ Bloco II } & 2.1 & 2 & 1 & 1 & 0 & 0,75 \\
\hline & 2.2 & 2 & 2 & 0 & 0 & 0,100 \\
\hline & 2.3 & 1 & 3 & 0 & 0 & 0,100 \\
\hline & 2.4 & 2 & 1 & 1 & 0 & 0,75 \\
\hline & 2.5 & 3 & 1 & 0 & 0 & 0,100 \\
\hline & 2.6 & 3 & 1 & 0 & 0 & 0,100 \\
\hline & 2.7 & 1 & 1 & 2 & 0 & 0,50 \\
\hline & 2.8 & 3 & 1 & 0 & 0 & 0,100 \\
\hline & 2.9 & 1 & 1 & 1 & 1 & 0,50 \\
\hline & 2.10 & 2 & 2 & 0 & 0 & 0,100 \\
\hline & 2.11 & 2 & 2 & 0 & 0 & 0,100 \\
\hline & 2.12 & 4 & 0 & 0 & 0 & 0,100 \\
\hline & Subtotal & 26 & 16 & 5 & 1 & - \\
\hline & $\begin{array}{l}\text { Percentual } \\
\text { por bloco }\end{array}$ & \multicolumn{5}{|c|}{$87,5 \%$} \\
\hline \multirow{7}{*}{ Bloco III } & 3.1 & 4 & 0 & 0 & 0 & 0,100 \\
\hline & 3.2 & 2 & 1 & 1 & 0 & 0,75 \\
\hline & 3.3 & 3 & 0 & 0 & 1 & 0,75 \\
\hline & 3.4 & 1 & 3 & 0 & 0 & 0,100 \\
\hline & 3.5 & 3 & 0 & 1 & 0 & 0,75 \\
\hline & Subtotal & 14 & 4 & 3 & 1 & - \\
\hline & $\begin{array}{l}\text { Percentual } \\
\text { por bloco }\end{array}$ & \multicolumn{5}{|c|}{$85 \%$} \\
\hline \multicolumn{2}{|c|}{ Total } & 48 & 27 & 9 & 2 & - \\
\hline Perce & al geral & \multicolumn{5}{|c|}{$87,5 \%$} \\
\hline
\end{tabular}

Fonte: Silva IS, et al., 2021. 
Tabela 5 - Tabela das respostas de juízes beneficiários por bloco de avaliação.

\begin{tabular}{|c|c|c|c|c|c|c|}
\hline \multirow[t]{2}{*}{ Blocos } & \multirow[t]{2}{*}{ Itens } & \multicolumn{4}{|c|}{$\begin{array}{c}\text { Escores }(\mathrm{N}=04) \\
\text { Escore } \mathrm{x} 100 / \mathrm{TA}+\mathrm{A}+\mathrm{PA}+\mathrm{l}= \\
\text { Percentual por escore }\end{array}$} & \multirow[t]{2}{*}{$\begin{array}{c}\text { Índice de } \\
\text { concordância por } \\
\text { item TA+A X } \\
\text { 100/Total de juízes }\end{array}$} \\
\hline & & TA & A & PA & I & \\
\hline \multirow{5}{*}{ Bloco I } & 1.1 & 3 & 1 & 0 & 0 & 0,100 \\
\hline & 1.2 & 2 & 2 & 0 & 0 & 0,100 \\
\hline & 1.3 & 3 & 1 & 0 & 0 & 0,100 \\
\hline & Subtotal & 8 & 4 & 0 & 0 & - \\
\hline & Percentual por bloco & \multicolumn{4}{|c|}{$100 \%$} & \\
\hline \multirow{8}{*}{ Bloco II } & 2.1 & 3 & 1 & 0 & 0 & 0,100 \\
\hline & 2.2 & 3 & 1 & 0 & 0 & 0,100 \\
\hline & 2.3 & 3 & 1 & 0 & 0 & 0,100 \\
\hline & 2.4 & 3 & 1 & 0 & 0 & 0,100 \\
\hline & 2.5 & 3 & 1 & 0 & 0 & 0,100 \\
\hline & 2.6 & 3 & 1 & 0 & 0 & 0,100 \\
\hline & Subtotal & 18 & 6 & 0 & 0 & - \\
\hline & Percentual por bloco & \multirow{2}{*}{\multicolumn{4}{|c|}{$100 \%$}} & \\
\hline \multirow{7}{*}{ Bloco III } & 3.1 & & & & 0 & 0,100 \\
\hline & 3.2 & 3 & 1 & 0 & 0 & 0,100 \\
\hline & 3.3 & 3 & 1 & 0 & 0 & 0,100 \\
\hline & 3.4 & 3 & 1 & 0 & 0 & 0,100 \\
\hline & 3.5 & 3 & 1 & 0 & 0 & 0,100 \\
\hline & Subtotal & 15 & 5 & 0 & 0 & - \\
\hline & Percentual por bloco & \multicolumn{4}{|c|}{$100 \%$} & \\
\hline \multirow{6}{*}{ Bloco IV } & 4.1 & 3 & 1 & 0 & 0 & 0,100 \\
\hline & 4.2 & 3 & 1 & 0 & 0 & 0,100 \\
\hline & 4.3 & 3 & 1 & 0 & 0 & 0,100 \\
\hline & 4.4 & 3 & 1 & 0 & 0 & 0,100 \\
\hline & Subtotal & 12 & 4 & 0 & 0 & - \\
\hline & Percentual por bloco & \multicolumn{4}{|c|}{$100 \%$} & \\
\hline \multirow{10}{*}{ Bloco V } & 5.1 & 3 & 1 & 0 & 0 & 0,100 \\
\hline & 5.2 & 3 & 1 & 0 & 0 & 0,100 \\
\hline & 5.3 & 3 & 1 & 0 & 0 & 0,100 \\
\hline & 5.4 & 3 & 1 & 0 & 0 & 0,100 \\
\hline & 5.5 & 3 & 1 & 0 & 0 & 0,100 \\
\hline & 5.6 & 3 & 1 & 0 & 0 & 0,100 \\
\hline & 5.7 & 3 & 1 & 0 & 0 & 0,100 \\
\hline & Subtotal & 21 & 7 & 0 & 0 & - \\
\hline & Percentual por bloco & \multicolumn{4}{|c|}{$100 \%$} & \\
\hline & Total & 74 & 26 & 0 & 0 & - \\
\hline
\end{tabular}

Fonte: Silva IS, et al., 2021.

\section{DISCUSSÃO}

A decisão de produzir e validar uma TE voltada à promoção da saúde do trabalhador emergiu da necessidade de fundamentação cientifica para auxiliar no processo de educação em saúde voltado a este público, tendo em vista, que há uma escassez de conteúdo validado com foco em orientações para prevenção de LER/ DORT'S. Optou-se por desenvolver uma TE que atendesse essa demanda e que chegasse ao público-alvo de maneira didática e descontraída, para isso o processo de validação de tecnologia seguindo ritos e protocolos pré-existentes foi de fundamental importância, haja vista a responsabilidade de cada pesquisador em emitir informações fidedignas e com maior clareza possível, como informou Monteiro DS, et al. (2019) em seu estudo.

Conhecer o público a qual se destina a TE é algo imprescindível, uma vez que o material precisa estar adequado à realidade das pessoas que irão se beneficiar desta ferramenta. Carneiro RF, et al. (2016) 
destacou que a efetividade de uma TE varia de acordo com a forma como este material é distribuído, especialmente no tocante da linguagem, por interferir diretamente no diálogo entre o intermediador e o grupo beneficiário. Justamente por isso, os autores optaram por fazer um levantamento prévio ao público pesquisado, com o intuito de conhecer a realidade dos envolvidos e instigar quais assuntos estes teriam interesse que fossem abordados no material educativo.

Nesta ocasião, 16 colaboradores da área da produção da empresa parceira foram entrevistados, sendo o sexo masculino de ampla predominância, deste $31,25 \%$ não sabiam o que era LER e DORT's, apesar da média de tempo de serviço destes serem de 5 anos e 6 meses, outro dado instigante é o nível de instrução destes colaboradores, onde $75 \%$ tinham apenas o ensino médio. O que poderia se arrazoar com o estudo de Neto AXS (2017) onde informa que a preocupação com a saúde laboral é proporcional à escolaridade, ou seja, quanto menor o nível de instrução, menor o interesse em buscar meios de prevenção a doenças ocupacionais, por isso a necessidade de um material acessível e que chame a atenção dos mesmos.

No término do questionário, os participantes poderiam sugerir quais conteúdos queriam que estivesse presente na TE, e foi possível perceber a significativa aclamação por assuntos voltados a alongamentos e ergonomia, respectivamente $80 \%$ e $70 \%$. Fazer uma busca prévia para determinar os assuntos abordados na produção de um TE não é uma exclusividade vivenciada por nós, anteriormente Frota NM, et al. (2015) realizou um estudo de validação sobre punção venosa periférica no estado do Ceará no qual os assuntos expostos em sua ferramenta foi resultado de um busca em artigos, dissertações, teses e livros que abordavam procedimentos de enfermagem, práticas baseadas em evidência e dados da Infusion Nursing Society(INS), posteriormente, os dados foram compilados e serviu de fonte norteadora.

Algo similar ocorreu no estudo de Benevides JL, et al. (2016) onde os autores buscaram produzir e validar uma TE sobre cuidados com úlceras venosas a partir de uma pesquisa desenvolvida em cinco fases; diagnóstico situacional; revisões de literatura; elaboração das ilustrações, layout, design e textos; validação aparente e de conteúdo e cálculo do Índice de Legibilidade de Flesch (IF); e teste piloto. Nesta obra, a primeira fase foi destinada a aplicação de questionário a 51 pessoas com diagnóstico de úlcera venosa (UV) acompanhadas em um ambulatório de cirurgia vascular, onde continha questionamentos quanto características das úlceras, localização, tempo de existência e no final teria que responder subjetivamente quais os cuidados ele considerava importante para o tratamento de úlceras.

A identificação desses temas serviu de base para a segunda fase, onde ocorreu o levanto bibliográfico em bases de dados que posteriormente o conteúdo gerado, proporcionou o embasamento da versão do material educativo. Sendo este desenho metodológico o mais semelhante da nossa pesquisa, considerando que ainda é raro trabalhos de validações voltados a saúde do trabalhador. Seguindo o processo metodológico, observase a composição da segunda amostra montada a partir de critérios básicos de inclusão e exclusão para profissionais de saúde participarem do grupo de juízes especialistas, contendo, 4 Fisioterapeutas do sexo masculino, com média de 7 anos e 5 meses de formação e 4 anos de experiência comprovada em saúde do trabalhador. A opinião desses profissionais foi de suma importância para evitar imprecisões técnicas e assim suas colocações no processo de validação foram acatadas e incluídas na versão final da TE, assim como destacou (MONTEIRO DS, et al., 2019).

Tanto a participação dos juízes especialistas quanto dos juízes beneficiários foi viabilizada pela escala adaptada de Likert que é uma escala comumente usada para aferir a concordância de pessoas a determinadas afirmações relacionadas a construtos de interesses. Consiste em tomar um construto e desenvolver uns conjuntos de afirmações relacionados à sua definição, para quais os envolvidos emitiram o seu grau de concordância (COSTA FJ, 2017). Esses participantes realizaram suas análises em instrumento dividido em blocos temáticos com opções de respostas divididas em quatro níveis. Após a aplicação desta escala, buscou-se aplicabilidade do rigor recomendado no processo de validação, para que todas as etapas e exigências fossem realizadas com êxito, com orienta a literatura (BRASIL GB, et al., 2018).

Vale destacar que no entendimento dos juízes a TE proposta atendeu as exigências e expectativas em torno de sua produção, sendo necessária somente uma rodada para atingir o IVC mínimo de concordância, 
muito em razão da agilidade e síntese gerada pela associação da técnica de Delfin ao processo metodológico da validação, que possibilitou impor um limite no número de ciclo de execução, para a busca da concordância. Nesse caso, a "concordância" representa o nível igual ou superior a de 70\% conseguido na última etapa da aplicação dos questionários determinada pelo pesquisador como sugerido no estudo de (NASCIMENTO MHM, 2012).

Os resultados mostraram alto grau de concordância nos itens avaliados, tanto nos juízes especialistas com IVC global de $87,5 \%$ quanto nos beneficiários com IVC global de 100\%. Observa-se maior aceitação por parte dos juízes beneficiários, os quais conferiram maior de concordância intergrupo na alternativa totalmente adequada e adequada. Este fenômeno também foi evidenciado no estudo de Monteiro DS, et al. (2019) que validou uma tecnologia educacional em biossegurança na atenção primária, a qual também foi obtida maior aceitação por parte do público-alvo. Em sumula, os autores acreditam que o grau de formação de cada grupo interfere diretamente na proporção de exigência quanto aos itens por eles avaliados.

De maneira geral, a maioria das respostas dos juízes foi concordante, e as insatisfações geradas na primeira versão do instrumento foram revisadas na segunda versão. No entanto, isso não interferiu na obtenção de altos índices de IVC. O que corroborou com outros estudos metodológicos de desenvolvimentos de tecnologias educativas que também validaram seus produtos com alto índice estatísticos como; a cartilha educativa para promoção da alimentação saudável entre pacientes diabéticos de autoria de Gonçalves MS, et al. (2019) que obteve IVC global de 0,96 e a tecnologia educativa para os cuidadores de pacientes submetidos a traqueostomia desenvolvida por Abreu ACS, et al. (2019) com IVC superior a 0,80 que era o esperado.

É comum durante o processo de validação, a adequação do material conforme a sugestão dos juízes, e isso mostra o engajamento recíproco entre pesquisadores e pesquisados a fim de entregar o instrumento mais aperfeiçoado possível, ao reunir diferentes pontos de vista sobre um mesmo instrumento. Destaca-se aqui em especial a atuação dos juízes especialistas que com suas contribuições técnicas enriquecem o material de conhecimento cientifico. Como enfatizou Gonçalves MS, et al. (2019) que os ajustes até a versão final validada possibilita a produção de um material altamente qualificado.

É importante frisar, que mesmo diante de inúmeras dificuldades na execução da pesquisa, a exemplo de conflitos de horários, número reduzido de profissionais com experiência em saúde do trabalhador e questões de ordem burocráticas, as limitações foram ultrapassadas e a validação foi realizada em sua plenitude, seguindo todas as fases e regras pré-existentes. Outra barreira de extrema limitação vivenciada pelos pesquisadores foi às repercussões da pandemia do covid-19, o que tornou necessário o isolamento social e forçou a adequação da nossa coleta de dados. Influenciando diretamente no número de amostra, sobretudo no de juízes beneficiários, reduzidos maciçamente pelas férias antecipadas ou demissões em massa.

Apesar disso, os autores buscaram se adaptar a nova realidade e conseguiram intermediar a execução de um processo de validação de boa qualidade e de possível reprodução, mesmo em plena pandemia. $O$ presente estudo apresenta limitações no tocante de reduzidas literaturas que abordem a temática, dificultando o diálogo e a interação com outros estudos, uma vez que a maioria dos artigos de validação é voltada as atribuições da enfermagem e praticamente nada é encontrado a respeito da Fisioterapia na saúde do trabalhador. O que possibilita o desbravamento do profissional Fisioterapeuta nesta linha de pesquisa, propiciando novas perceptivas para o acervo literário das produções de TE.

\section{CONCLUSÃO}

Desta forma, concluímos que a TE produzida neste estudo é válida e apropriada para utilização de profissionais Fisioterapeutas e afins no processo de educação em saúde, as análises com alto índice de concordância entre os dois grupos de juízes reconhecem a objetividade, a pertinência do conteúdo e a facilidade de comunicação com o público-alvo, tornando está diferente de tantas outras TE, alcançando assim o objetivo a qual se propõe. Contudo, não é possível garantir a superioridade efetiva de um material validado para outro não validado, o que instiga o desdobramento de outros estudos afim de colocar em prática a avaliação da efetividade de TE validadas. 


\section{AGRADECIMENTOS E FINANCIAMENTO}

Agradecemos a todos os gestores e colaboradores da empresa envolvida na pesquisa, por propiciar a viabilidade de coleta de dados em seu estabelecimento, o apoio e a disponibilidade desta instituição foi de fundamental importância agregando valor ético e moral durante todo o processo. Aos Fisioterapeutas especialistas que se dispuseram a contribuir com o estudo, ultrapassando todas as limitações acerca de indisponibilidade de horário em prol da ciência.

\section{REFERÊNCIAS}

1. ABREU ACS, et al. Tecnologia Educativa para os Cuidados de Pacientes Submetidos a Traqueostomia: Estudo de Validação. Revista de Atenção à Saúde, 2019; 17(59):19-32.

2. BENEVIDES JL, et al. Development and validation feducational technology for venous ulcer care. Revista da Escola de Enfermagem da USP, 2016; 50(2): 306-312.

3. BRASIL GB, et al. Tecnologia educacional para pessoas que convivem com HIV: estudo de validação. Revista Brasileira de Enfermagem, 2018; 71(4): 1754-9.

4. CARNEIRO FR, et al. CARTILHA: um instrumento para melhora dos hábitos posturais de crianças e adolescentes. In: MACENA RHM, et al. Promoção da Saúde de Crianças e Adolescentes: Manual prático de ações para Fisioterapia, 2016; 1: 116-125.

5. COSTA FJ. Mensuração e desenvolvimento de escalas: Aplicação em administração. Revista de Administração de Empresas, 2017; 57: 97-98.

6. FROTA NM, et al. Validação De Hipermídia Educativa Sobre Punção Venosa Periférica. Texto Contexto Enfermagem, 2015; 24(2): 353-61.

7. GONÇALVES MS, et al. Construção e validação de cartilha educativa para promoção da alimentação saudável entre pacientes diabéticos. Revista Brasileira de Promoção à Saúde, 2019; 32: 7781.

8. HUGUE TD, PEREIRA JUNIOR A. Prevalência de dor osteomuscular entre os funcionários administrativos da Unifebe. Revista UNIFEB Santa Catarina, 2011; 9: 1-9.

9. MELO IA. Validação de um manual educativo como tecnologia de enfermagem para pessoas com Diabetes Mellitus tipo 2. Aracaju -Programa de pós-graduação em enfermagem - Universidade Federal de Sergipe, 2017.

10. MONTEIRO DS, et al. Validação de uma tecnologia educativa em biossegurança na atenção primária. Revista Cuidarte, 2019; 10(2): e654.

11. MOREIRA AP, et al. Jogo educativo de administração de medicamentos: um estudo de validação. Revista Brasileira de Enfermagem. Brasília, DF, 2014; 67(4): 528- 34.

12. MOSER AD, KERHIG R. O conceito de saúde e seus desdobramentos nas várias formas de atenção à saúde do trabalhador. Revista Fisioterapia em Movimento, Curitiba. 2006; 19 (4): 89-97.

13. NASCIMENTO MHM. Tecnologia para mediar o cuidar-educando no acolhimento de "familiares cangurus" em unidade neonatal: Estudo de Validação. Dissertação (Mestrado em Enfermagem) - Centro de Ciências Biológicas e da Saúde. Universidade do Estado do Pará, Belém, 2012; 30-31p.

14. NETO AXS. A importância da prática da ginástica laboral com funcionários da Universidade Federal do Rio Grande do Norte na prevenção da LER/DORT. 2017. Trabalho de Conclusão de Curso. Universidade Federal do Rio Grande do Norte, Natal, 2017;21-37p.

15. NIETSCHE EA, et al. Tecnologias inovadoras do cuidado em enfermagem. Revista Enfermagem UFSM, $2012 ; 2$ (1): 182-189.

16. Polit DF, Beck CT, Hungler BP. Fundamentos de pesquisa em enfermagem: métodos, avaliação e utilização. 5. ed. Porto Alegre: Artmed; 2004.

17. SERRA MVGHB, et al. Ginástica laboral: uma revisão de literatura. Revista Pesquisa em Fisioterapia, $2014 ; 4$ (3): 197-205.

18. SILVA LPS, MORSCH P. Os benefícios da fisioterapia nas doenças osteomusculares associadas ao trabalho. Revista da Faculdade de Educação e Meio Ambiente (FAEMA), Ariquemes, 2019; 10 (1): 182-190.

19. SILVA WR, et al. Programa de intervenção fisioterapêutica na prevenção de LER/DORT em uma instituição pública do sertão paraibano. Revista Temas em Saúde. João Pessoa, PB. 2016; 460- 486.

20. SOUSA CS, TURRINI RNT. Validação de constructo de tecnologia educativa para pacientes mediante aplicação de técnica Delphi. Revista Acta Paulista Enfermagem, 2012; 25 (6): 990-996. 